\title{
Auszeichnungen für Dr. Thomas Wiesner und Dr. Amaya Virós
}

Jährlich vergibt die LEO Pharma Research Foundation zwei Preise an herausragende und außergewöhnlich befähigte Wissenschaftler. In diesem Jahr geht der mit 1 Mio. Dänischen Kronen (DKK) dotierte Gold Award an Dr. Amaya Virós, und der mit 500.000 DKK dotierte Silver Award an Dr. Thomas Wiesner.

ZurEhrung und Förderung bedeutender Leistungen in der Gesundheitsforschung hat die LEO Pharma Research Foundation im Jahr 2008 Auszeichnungen in Gold und Silber ausgerufen. Die Europäische Gesellschaft für Dermatologische Forschung (ESDR) schlägt Kandidaten vor, und die LEO Pharma Research Foundation wählt die Preisträger aus.

Dr. Thomas Wiesner beschritt neue Wege auf der Suche nach kausal wirksamen Krebstherapien. Wiesner dissertierte zu genomischen Aberrationen von kutanen Lymphomen und absolvierte seine Ausbildung als Facharzt für Dermatologie an der Medizinischen Universität Graz. Wiesner war fünf Jahre lang Leiter für grundlegende und translationale Forschung. Er arbeitet an der Kombination von Hochdurchsatz-Sequenziertechnologien, computergestützten Ansätzen und funktionellen Untersuchungen, um die relevanten genomischen und epigenomischen Aberrationen bei Hautkrebs $z u$ bestimmen und Wege für neue Behandlungsoptionen zu bahnen.

Dr. Amaya Virós beschrieb Mechanismen bei der Entwicklung von Plat- tenepithelkarzinomen und Melanomen. Virós wurde kürzlich ein Wellcome Trust Intermediate Clinician Scientist Fellowship am Cancer Research UK Manchester Institute, UK, gewährt. Virós will sich auch in Zukunft mit kaum erforschten Fragen im Bereich Hautkrebs und Alterung beschäftigen. mitteilung der LEO

PHARMA Research

Foundation

Hier steht eine Anzeige. 